\title{
Rehabilitation Including Manual Therapy Management Following Complicated Immobilized Radial Head Fracture and Elbow Stiffness: A Case Report
}

\author{
M. D. Stump"1,2, P. S. Sizer ${ }^{2,3}$, J. M. Brismée ${ }^{2,3}$ \\ ${ }^{1}$ George Erb Physical Therapy Inc., Camarillo, USA \\ ${ }^{2}$ International Academy of Orthopedic Medicine-United States, Tucson, USA \\ ${ }^{3}$ Center for Rehabilitation Research, Texas Tech University Health Sciences Center, Lubbock, USA \\ Email: jm.brismee@ttuhsc.edu
}

Received 7 February 2014; revised 6 March 2014; accepted 5 April 2014

Copyright (C) 2014 by authors and Scientific Research Publishing Inc.

This work is licensed under the Creative Commons Attribution International License (CC BY). http://creativecommons.org/licenses/by/4.0/

(c) (i) Open Access

\begin{abstract}
Radial head fractures are one of the most common fractures of the elbow and typically occur after a fall on an outstretched arm. When the fracture is displaced, unfavorable outcomes can occur and lead to early arthritis. Additionally, elbow pain and stiffness due to immobilization or surgical reduction and stabilization typically ensues and can limit one's overall upper extremity function. Rehabilitation following such a fracture can be challenging and the inclusion of manual therapy in the management process has not been described to date in the literature. Therefore, the aim of this case report is to present the evaluation and treatment of a 40 -year-old female following a complicated radial head fracture. The rehabilitation conducted over a four-month course is discussed and the benefits of joint specific manual therapy in the rehabilitation are highlighted.
\end{abstract}

\section{Keywords}

Radius Fracture, Musculoskeletal Manipulations, Rehabilitation, Elbow, Contracture

\section{Introduction}

Radial head fractures are one of the most common fractures of the elbow [1]-[4] and typically occur after a fall on an outstretched arm [5]. Research has suggested that non-displaced fractures have minimal residual deficits; however, displaced fractures tend to display a non-favorable outcome even if short-term follow-up does not re- 
veal functional deficits. Residual post-traumatic joint incongruity may lead to elbow osteoarthritis [5]. Additionally, it is well documented that elbow stiffness ensues following elbow fracture [2] [6]-[8]. Outcome research on rehabilitation following elbow fracture treated conservatively or surgically is limited although most protocols recommend both active and passive range of motion (ROM). Additionally, the role has been established for dynamic splinting to restore elbow ROM [9]-[13]. However, the benefits of dynamic splinting are most notable in the first few months following injury or surgery, though gains can been observed up to 12 months [9]. Gelinas et al. [14] reported on the effectiveness of turnbuckle splinting; however, in their protocol the subjects wore the splint for 20 hours per day and were allowed to remove the splint for one hour periods of free movement at breakfast, lunch, dinner and before bed. The time commitment for splinting is not always feasible. Furthermore, depending on one's insurance the splint can be cost prohibitive. Unfortunately, the literature establishing the role and benefit of joint specific treatment is lacking [1]-[3] [6] [7] [15]. This case report presents the examination findings of a post-traumatic malunion radial head fracture with subsequent open reduction internal fixation (ORIF), the interpretation of the examination findings, interventions used during the rehabilitation process, and the outcomes of treatment. More specifically, this case report highlights the addition of joint specific manual intervention to the patient management to restore mobility and full elbow extension without the prolonged use of dynamic splinting in a patient with complicated radial head fracture.

\section{Case Report}

A right handed 40-year-old hair stylist was referred to physical therapy for evaluation and treatment of a radial head malunion fracture status-post ORIF secondary to a fall while roller skating ten weeks prior. Upon injury, she landed on her buttocks and right elbow. She went to the local urgent care initially for evaluation. Radiographs were taken at that time, but she was not informed she had sustained a radial head fracture. About one week later, she was contacted by the urgent care and was directed to pick up her radiographs and to follow-up with an orthopedist. As she was not advised on the nature of her injury, she casually scheduled an appointment with an orthopedist and, after his evaluation, decided she wanted a second opinion. Her operating surgeon evaluated her three to four weeks after her injury. She was formally diagnosed with a radial head fracture by both physicians, but unfortunately, the fracture had begun to heal by the time she was evaluated by the second orthopedist and her fracture was classified as a malunion radial head fracture, which required reduction (Figure 1(a), Figure 1(b)). She underwent surgery to correct the defect eight weeks after initial injury with a manipulation and ORIF and wore a cast for approximately one week post-op. The cast was removed five days prior to her physical therapy evaluation.

Following cast removal, the patient had begun to use her arm for simple activities but reported significant stiffness and moderate ache in her right elbow, forearm, wrist, and hand. The patient reported increased symptoms in the morning with mild increase in flexibility as the day progressed; however, the stiffness and soreness were consistent throughout the day. At the time of her initial evaluation, she had been directed by her surgeon to limit her lifting to less than one pound. She reported difficulties with bathing and self-care tasks due to her ROM limits and weakness. She was unable to use the right upper extremity for most activities of daily living (ADL's) and lifestyle tasks such as putting make-up on, cooking, cleaning and laundry. Additionally, she was unable to perform her work as a hairstylist as it required lifting greater than one pound and more elbow flexion and extension than was available. Her primary complaints at the time of the initial evaluation were: significant restriction in ROM, weakness in the upper extremity, and increased pain. She rated her pain on a 10-point scale as 7/10 at worst, $4 / 10$ at best, and $4 / 10$ at the time of the initial evaluation. She stated that she felt a dull, achy type pain most of the time but did experience episodes of sharp pain. This patient's goal was to regain normal use of her right arm for her work and lifestyle activities.

Additional information gathered included the use of thyroid medication for hypothyroidism, pain and anti-inflammatory medications for the elbow symptoms at the time of her evaluation. She did not have any contraindications to begin physical therapy, and her prescription for physical therapy included instructions for full AROM and gentle PROM, so a modified elbow examination was completed.

\subsection{Clinical Examination}

The patient was evaluated two weeks following ORIF, so the initial examination (Table 1) was limited due to the surgical precautions and prescription limitations. She presented with a $1.5 \mathrm{~cm}$ increase in swelling about the 


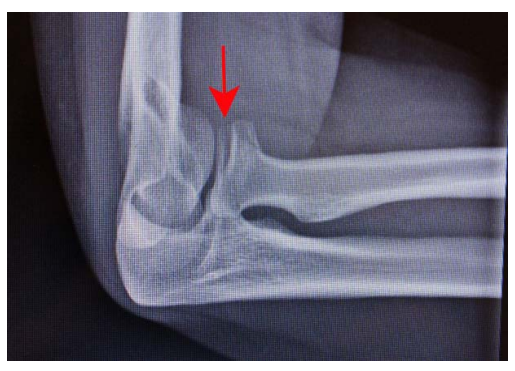

(a)

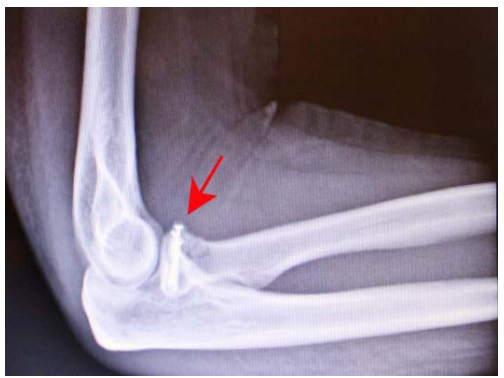

(c)

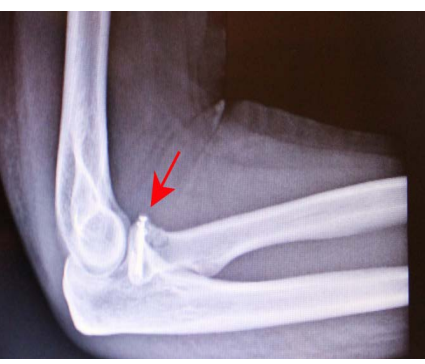

(b)

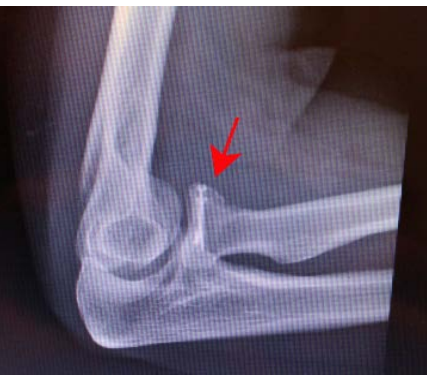

(d)

Figure 1. (a) (b): Pre- and post-operative radiographs. (a) shows the radial head fracture (red arrow). (b) shows the radial head post reduction with instrumentation (red arrow). (c) (d): Post-operative radiographs showing preand post-radial head displacement. (c) shows the radial head post reduction with instrumentation (red arrow). (d) shows the radial head displacement of < $1 \mathrm{~mm}$ (red arrow) found at the eight-week post-operative visit.

Table 1. Outcomes of the Initial Physical Examination.

\begin{tabular}{ccc}
\hline \multicolumn{2}{c}{ Inspection-Healthy appearing female in no apparent distress. Steri-strips over incision. } \\
\hline $\begin{array}{c}\text { Girth Measurement: Elbow flexion } \\
\text { crease }\end{array}$ & Right & Left \\
& $25.5 \mathrm{~cm}$ & $23 \mathrm{~cm}$ \\
Elbow AROM & Range of Motion & Left \\
Extension & $-60^{\circ}$ & $4^{\circ}$ \\
Flexion & $90^{\circ}$ & $145^{\circ}$ \\
Supination & $45^{\circ}$ & $90^{\circ}$ \\
Pronation & $60^{\circ}$ & $85^{\circ}$ \\
& & \\
Elbow PROM & Right & $\mathbf{L e f t}^{\circ}$ \\
Extension & $-35^{\circ}$, end feel - Firm & $5^{\circ}$ \\
Flexion & $95^{\circ}$, end feel - Firm & $150^{\circ}$ \\
Supination & $65^{\circ}$, end feel - Firm & $90^{\circ}$ \\
Pronation & $80^{\circ}$, end feel - Firm & $90^{\circ}$ \\
Strength & Not Tested due to surgical precautions & Not Tested due to surgical precautions
\end{tabular}

Palpation: Palpable tenderness and increased muscular tone noted. Skin is warm to touch and painful along the area of the radial head.

right elbow crease as compared to the left elbow crease. Her score on the Disabilities of Arm, Shoulder, Hand (DASH) was 60/100, and her score on the DASH Work Module was 75\% limited. Muscle testing was not performed during the initial evaluation due to surgical precautions, and due to the post-surgical nature of her injury, special tests were not required either. Increased skin temperature and tenderness to palpation were noted about the radial head and incision. Minimal scar tissue thickening was noted. 


\subsection{Interpretation of Clinical Examination Findings and Diagnosis}

Based on the examination findings, the patient presented with a non-capsular pattern of limitation status post immobilization. On examination, she demonstrated a limit of motion into elbow flexion, extension, supination, and pronation. The capsular pattern of limitation for the elbow has been shown to be flexion limited much more than extension [16]. This patient had significant limitations of motion into both flexion and extension with a firm end-feel at the end-range of both flexion and extension, and her limitation was found during active and passive ROM. Given that she underwent surgery with immobilization status post a traumatic injury, it was felt that the large limitation of motion was due to the surgery and immobilization rather than due to an actual degenerative process in the elbow, thus, a non-capsular pattern of limitation due to the immobilization was considered as the primary physical therapy diagnosis.

\subsection{Prognosis and Plan of Care}

The prognosis for this patient was good. She was relatively young and healthy and highly motivated to return to her active lifestyle. However, due to the elbow tendency for stiffness [2] [6] [7] following a traumatic injury, and due to the surgical intervention following a malunion fracture, there was a significant probability she would not regain full elbow range of motion and function. However, the physical therapy expectation was that she would gain functional mobility of the elbow and be able to return to her prior level of activity without limitation. The primary goal was to allow her to return to her full work duties as a hair stylist. The patient was advised to order a Dynasplint as dynamic splinting [9] [14] has been shown to increase ROM in the stiff elbow, and a prescription was obtained from her surgeon.

\section{Interventions}

This patient was treated two times per week over the course of four months. During the first six to eight weeks, the physician's directives consisted of PROM and soft tissue management only as to allow adequate post- operative healing. She maintained a weight restriction of one to two pounds only. Additionally, the patient discontinued the use of the dynamic splinting due to her lack of progress and the out-of-pocket-cost she incurred while renting the unit. Gelinas et al. [14] suggested wearing the splint for 20 hours per day for approximately six months for an increase of $\geq 10^{\circ}$ of motion. At her eight week follow-up visit to her surgeon, repeated radiography revealed $<1 \mathrm{~mm}$ displacement of the radial head — the radial head was depressed with appropriate hardware placement (Figure 1(c), Figure 1(d)). Consequently, we were not able to progress PROM and were to continue with gentle PROM and soft tissue work. After an additional two weeks, the weight restrictions were lifted and we were able to progress the rehabilitation program. Manual interventions including soft tissue management and exercise were used to achieve her ROM goals. Passive ROM included elbow flexion, extension, supination and pronation. Joint specific mobilizations including humeroulnar joint extension traction and glides to improve endrange elbow extension were added to the treatment plan. The decision to include joint specific mobilizations was based on the outcome of joint play testing where a limitation was found with humeroulnar joint traction, medial, and lateral gliding. Joint specific manual techniques included: traction from a prepositioned elbow extension (Figure 2(a), medial gliding in prepositioned extension and pronation (Figure 2(b)), lateral gliding in prepositioned extension and pronation (Figure 2(c)), as well as end-range extension curved gliding (Figure 2(d)). Joint specific mobilizations were performed for 4 - 5 bouts of 40 seconds per mobilization [17] with an isometric contraction into extension following mobilization [18]. Exercises included general strengthening for the shoulder complex, biceps, triceps, and forearm supination and pronation.

\section{Outcomes}

This patient was treated over a protracted period of time due to the challenges faced during the healing process. After the PROM restrictions were lifted, she remained with limited terminal elbow extension, which was restored following the addition of joint specific humeroulnar mobilizations. At her last physical therapy visit, she achieved full elbow extension ( 0 degrees) and had returned to her full work duties as a hair stylist. She continued to deal with pain post exercise but was gradually returning to all prior activities without limitation. At the end of her treatment (Table 2) she rated her pain at 3/10 at worst, $0 / 10$ for current and best, and she described her symptoms as soreness in nature that only bothered her after exercise or extensive use of her right upper ex- 


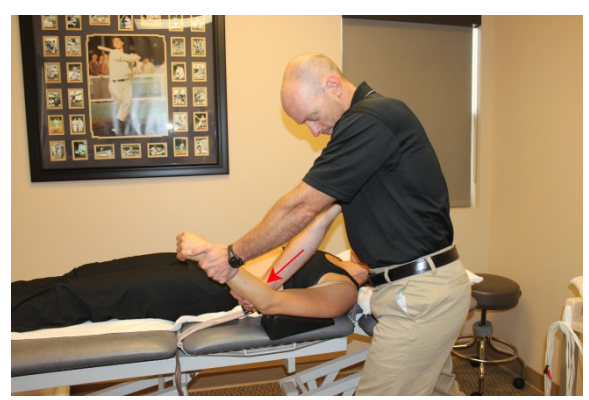

(a)
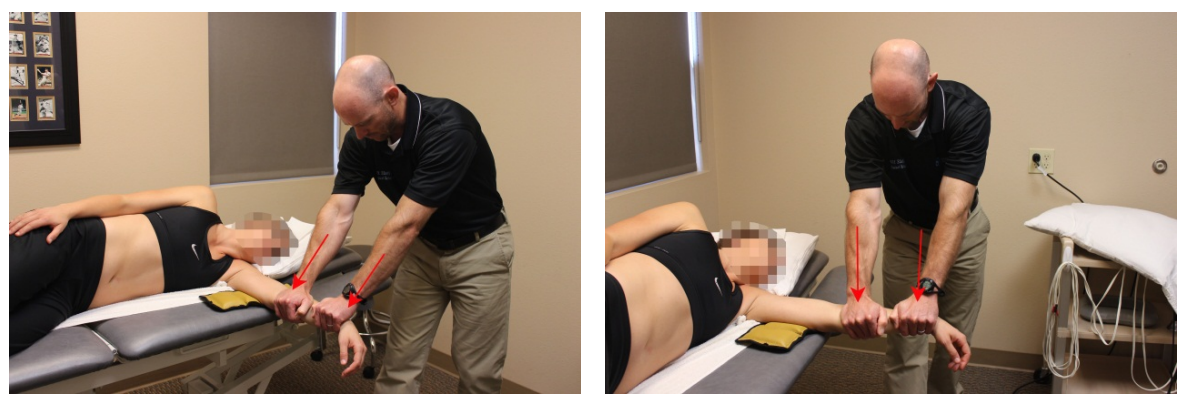

(b)

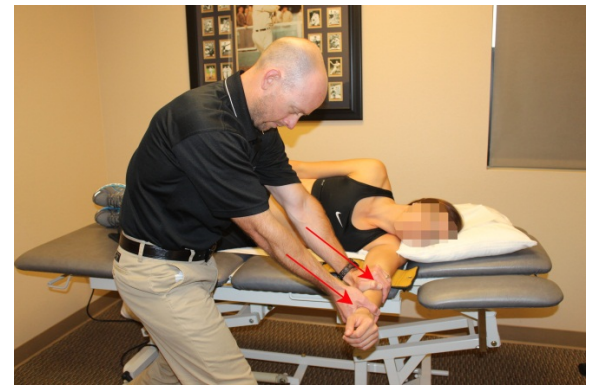

(c)
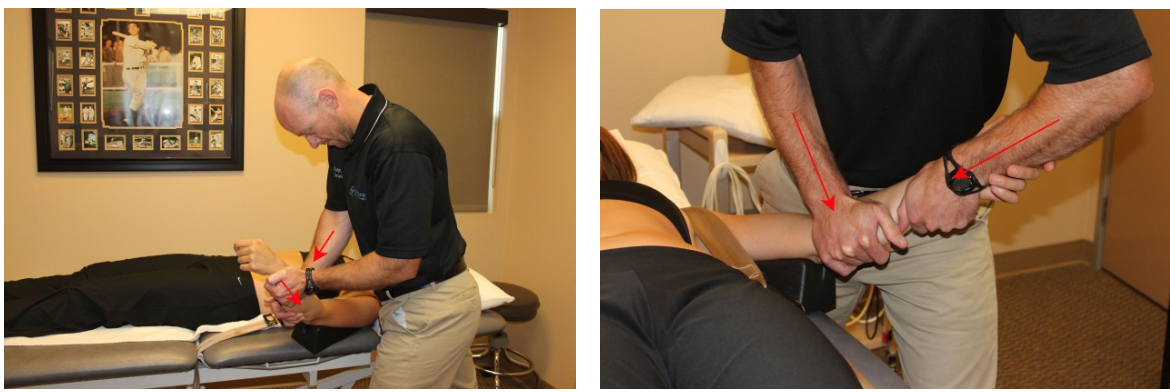

(d)

Figure 2. (a): Traction from prepositioned elbow extension. The humerus is fixed proximally and traction is applied through the proximal hand in a direction that is $45^{\circ}$ posterodistal to the ulna, and perpendicular to a line through both epicondyles (red arrow) while the therapists distal hand maintains the pronation. (b): Medial gliding in prepositioned extension and pronation. The direction of glide is performed at an angle of $60^{\circ}$ posteromedial from a line connecting both humeral epicondyles, and perpendicular to the ulna (red arrow). (c): Lateral gliding in prepositioned extension and pronation. The direction of glide is performed at an angle of $60^{\circ}$ posterolateral from a line connecting both humeral epicondyles and perpendicular to the ulna (red arrow). (d): End-range extension curved gliding. The therapist applies traction to the humerounlar joint with the proximal hand at $45^{\circ}$ posterolateral to the ulna, and perpendicular to a line through both of the epicondyles (red arrow). The distal hand glides the ulna posterosuperiorly (red arrow). Both movements are applied in equal proportion as the elbow is moved into terminal extension. 
Table 2. Outcomes of the Final Examination.

\begin{tabular}{|c|c|c|}
\hline \multicolumn{3}{|c|}{ Inspection—Healthy appearing female in no apparent distress. } \\
\hline $\begin{array}{c}\text { Girth Measurement: Elbow flexion } \\
\text { crease }\end{array}$ & $\begin{array}{c}\text { Right } \\
23.5 \mathrm{~cm}\end{array}$ & $\begin{array}{c}\text { Left } \\
23 \mathrm{~cm}\end{array}$ \\
\hline \multicolumn{3}{|c|}{ Range of Motion } \\
\hline Elbow AROM & Right & Left \\
\hline Extension & $0^{\circ}$ & $4^{\circ}$ \\
\hline Flexion & $120^{\circ}$ & $145^{\circ}$ \\
\hline Supination & $90^{\circ}$ & $90^{\circ}$ \\
\hline Pronation & $85^{\circ}$ & $85^{\circ}$ \\
\hline Elbow PROM & Right & Left \\
\hline Extension & $2^{\circ}$ Hyperextension & $5^{\circ}$ Hyperextension \\
\hline Flexion & $135^{\circ}$, end feel - firm & $150^{\circ}$ \\
\hline Supination & $90^{\circ}$, end feel - normal & $90^{\circ}$ \\
\hline Pronation & $90^{\circ}$, end feel - normal & $90^{\circ}$ \\
\hline Strength & Right & Left \\
\hline \multicolumn{3}{|l|}{ Shoulder: } \\
\hline ABD & $5 / 5$ & $5 / 5$ \\
\hline ADD & $5 / 5$ & $5 / 5$ \\
\hline IR & $5 / 5$ & $5 / 5$ \\
\hline ER & $4+/ 5$ & $4+/ 5$ \\
\hline \multicolumn{3}{|l|}{ Elbow: } \\
\hline Flexion & $4+/ 5$ & $5 / 5$ \\
\hline Extension & $4+/ 5$ & $5 / 5$ \\
\hline Supination & $5 / 5$ & $5 / 5$ \\
\hline Pronation & $5 / 5$ & $5 / 5$ \\
\hline \multicolumn{3}{|c|}{ Palpation: No palpable tenderness or increased muscular tone noted } \\
\hline
\end{tabular}

tremity. She denied pain to palpation and she had approximately $0.5 \mathrm{~cm}$ of swelling about the elbow crease. Her DASH score improved from a 60/100 to a 66.6/100 and her work module improved from $75 \%$ limited to $12.5 \%$ limitation.

\section{Discussion}

This is the first case study to report favorable outcome for a patient following complicated radial head fracture treated surgically with ORIF using physical therapy management including the use of joint mobilizations specific to the humero-ulnar joint on elbow ROM and function. This patient sustained a malunion radial head fracture treated surgically with a manipulation and a screw fixation, and an observable radial head displacement at her eight-week post-operative appointment. Displaced radial head fractures are known to be challenging to manage with more than $50 \%$ of the cases displaying unfavorable outcomes including arthrofibrosis [19]. Therefore, joint mobilizations were used without the prolonged use of dynamic splinting for the recovery of the patient's elbow extension. This patient did not see the value in the dynamic splinting compared to the cost, and, consequently, she discontinued its use in less than two months. According to Gelina et al. [14], turnbuckle splinting was beneficial in restoring ROM when worn for 20 hours per day for up to six months. Lindenhovius et al. [9], in a study comparing static progressive stretching (turnbuckle splinting) and dynamic splinting reported no significant differences between the two over the course of six months to one year, but stated that patience is warranted. In their study, the subjects wore the splints per the manufacturer's recommendations, which, for the dynamic splinting, was six to eight hours per night [9]. This patient did not follow the recommendations; she wore the splint for up to four hours per night, on average, due to complaints of pain as well as an inability to sleep.

Patients with orthopedic conditions, specifically following surgical procedures, are routinely referred to physical therapy for rehabilitation services. Radial head fractures have been reported to account for 33\% of fractures 
around the elbow [2]. The typical rehabilitation protocol for both conservative and surgical treatments consider PROM as an integral procedure but seldom reflect on the benefit of manual therapy, specifically joint specific mobilizations. Despite literatures' confirmation of the tendency for elbow stiffness following trauma and surgery [2] [6] [7] research on the benefits of joint mobilizations for restoring humeroulnar joint mobility is lacking and needs further delineation. Instead, the literature suggests procedures such as manipulation under anesthesia, bracing, and open or arthroscopic release as primary treatment options for resolving post traumatic elbow stiffness, which have been shown to have success in relieving elbow contracture [7] [10] [11] [20]-[24]. However, not all the options are feasible for patients for various reasons such as the length of time required for recovery or wearing a splint, cost of another procedure or renting a splinting device, or simply due to the business of life. One report by Bano and Kahlon [1] addressed the role of manual therapy during rehabilitation of elbow fractures. The authors suggested that physical therapists who are highly competent and experienced in manual techniques, may begin joint specific mobilization techniques at 6 weeks post-operatively even following traumatic fractures [1].

In this patient, full elbow extension ROM was achieved neither with PROM procedures alone, nor with the two-month use of dynamic splinting. The benefits of PROM were exhausted and we did not measure an improvement of active extension past a $10^{\circ}$ limit until the addition of joint specific manual therapy was introduced into her plan of care (Table 3). In her last month of therapy, when joint specific mobilizations were introduced in the plan of care, she achieved $2^{\circ}$ of elbow hyperextension (she had $5^{\circ}$ of hyperextension with left elbow extension) within eight visits. Her previous three months of therapy, which included soft tissue mobilizations, PROM, and dynamic splinting were unable to garner the ROM she wanted to achieve and required for a full return to her work duties and lifestyle activities. Through the addition of joint specific mobilizations we were able to recapture her needed ROM. Thus, the above case report sheds light on the benefits of the addition of joint specific treatment in addition to PROM to restore elbow extension without manipulation under anesthesia, arthroscopic capsular release, or prolonged bracing. This case was protracted due to initial malunion, manipulation under anesthesia, radial head re-fracture with screw fixation, and the radial head displacement. After the restrictions were lifted and the physical therapist was allowed to perform more than general PROM, the patient began to regain terminal elbow extension through the addition of joint specific mobilizations for restoring elbow extension. She was able to return to full work duties and routine ADL's only after the onset of joint specific mobilizations to restore full elbow extension. She was likely challenged by joint limitations that were resolved through targeted joint mobilizations aimed at restoring capsular mobility. Interestingly, this patient fractured her radial head, but her limitation of motion was found in the humeroulnar joint post immobilization. This is likely due to the large elbow capsule that encloses the humeroulnar and the radiohumeral joints; therefore, following joint play testing for the involved joints, joint specific mobilizations were introduced into the plan of care. The specific joint mobilizations used were targeted to stretch the elbow capsule and inert tissues. Stretching the elbow capsule in a specific fashion allowed elbow motion restoration that was unachievable through PROM alone. Future research should consider the added benefit of joint specific mobilizations for full restoration of elbow complex ROM, especially in the presence of a complicated fracture.

\section{Conclusion}

Radial head fractures are one of the most common fractures of the elbow, and, when the fracture is displaced, can result in unfavorable outcomes leading to early arthritis and limited ROM. This reported case presented the

\begin{tabular}{ccc} 
Table 3. Changes in ROM. & & \\
\hline & Elbow Extension - Active & Elbow Extension - Passive \\
\hline Initial Examination & $-60^{\circ}$ & $-35^{\circ}$ \\
1 month & $-25^{\circ}$ & $-20^{\circ}$ \\
2 months & $-15^{\circ}$ & $-10^{\circ}$ \\
3 months & $-10^{\circ}$ & $-7^{\circ}$ \\
4 months(Discharge) & $0^{\circ}$ & $2^{\circ}$ hyperextension \\
\hline
\end{tabular}


successful management of a 40-year-old female following a complicated radial head fracture. The rehabilitation was conducted over a four-month period with the inclusion of joint specific manual therapy to recapture elbow range of motion, specifically elbow extension, which was unattainable through PROM or dynamic splinting alone.

\section{References}

[1] Bano, K.Y. and Kahlon, R.S. (2006) Radial Head Fractures-Advanced Techniques in Surgical Management and Rehabilitation. Journal of Hand Therapy, 19, 114-136. http://dx.doi.org/10.1197/j.jht.2006.02.011

[2] Liow, R.Y.L., Cregan, A., Nanda, R. and Montgomery, R.J. (2002) Early Mobilisation for Minimally Displaced Radial Head Fractures Is Desirable: A Prospective Randomised Study of Two Protocols. Injury, 33, 801-806. http://dx.doi.org/10.1016/S0020-1383(02)00164-X

[3] Paschos, N.K., Mitsionis, G.I., Vasiliadis, H.S. and Georgoulis, A.D (2013) Comparison of Early Mobilization Protocols in Radial Head Fractures. Journal of Orthopaedic Trauma, 27, 134-139. http://dx.doi.org/10.1097/BOT.0b013e31825cf765

[4] Pike, J.M., Athwal, G.S., Faber, K.J. and King, G.J.W. (2009) Radial Head Fractures-An Update. The Journal of Hand Surgery, 34, 557-565. http://dx.doi.org/10.1016/j.jhsa.2008.12.024

[5] Herbertsson, P., Josefsson, P.-O., Hasserius, R., Karlsson, C., Besjakov, J. and Karlsson, M. (2004) Uncomplicated Mason Type-II and III Fractures of the Radial Head and Neck in Adults: A Long-Term Follow-Up Study. The Journal of Bone \& Joint Surgery, 86, 569-574.

[6] Harding, P., Rasekaba, T., Smirneos, L., and Holland, A.E. (2011) Early Mobilisation for Elbow Fractures in Adults. In: The Cochrane Collaboration and P. Harding, Eds., Cochrane Database of Systematic Reviews, John Wiley \& Sons, Ltd., Chichester.

[7] Koh, K.H., Lim, T.K., Lee, H.I. and Park, M.J. (2013) Surgical Release of Elbow Stiffness after Internal Fixation of Intercondylar Fracture of the Distal Humerus. Journal of Shoulder and Elbow Surgery, 22, 268-274. http://dx.doi.org/10.1016/j.jse.2012.10.024

[8] Søjbjerg, J.O. (1996) The Stiff Elbow. Acta Orthopaedica Scandinavica, 67, 626-631. http://dx.doi.org/10.3109/17453679608997771

[9] Lindenhovius, A.L.C., Doornberg, J.N., Brouwer, K.M., Jupiter, J.B., Mudgal, C.S. and Ring, D. (2012) A Prospective Randomized Controlled Trial of Dynamic versus Static Progressive Elbow Splinting for Posttraumatic Elbow Stiffness. The Journal of Bone \& Joint Surgery, 94, 94-700.

[10] King, G.J.W. and Faber, K.J. (2000) Post Traumatic Elbow Stiffness. Orthopedic Clinics of North America, 31, 129143. http://dx.doi.org/10.1016/S0030-5898(05)70133-4

[11] Doornberg, J.N., Ring, D. and Jupiter, J.B. (2006) Static Progressive Splinting for Posttraumatic Elbow Stiffness. Journal of Orthopaedic Trauma, 20, 400-404. http://dx.doi.org/10.1097/00005131-200607000-00006

[12] Keschner, M.T. and Paksima, N. (2007) The Stiff Elbow. Bulletin of the NYU Hospital for Joint Diseases, 65, 24-28.

[13] Issack, P.S. and Egol, K.A. (2006) Posttraumatic Contracture of the Elbow: Current Management Issues. Bulletin of the NYU Hospital for Joint Diseases, 63, 129-136.

[14] Gelinas, J.J., Faber, K.J., Patterson, S.D. and King, G.J.W. (2000) The Effectiveness of Turnbuckle Splinting for Elbow Contractures. The Journal of Bone \& Joint Surgery, 82-B, 74-78. http://dx.doi.org/10.1302/0301-620X.82B1.9792

[15] Mathew, P.K., Athwal, G.S. and King, G.J. (2009) Terrible Triad Injury of the Elbow: Current Concepts. Journal of the American Academy of Orthopaedic Surgeons, 17, 137-151.

[16] Winkel, D., Matthijis, O. and Phelps, V. (1997) Diagnosis and Treatment of the Upper Extremities: Nonoperative Orthopaedic Medicine and Manual Therapy. Aspen, Gaithersburg.

[17] Taylor, D.C., Dalton Jr., J.D., Seaber, A.V. and Garrett Jr., W.E. (1990) Viscoelastic Properties of Muscle-Tendon Units. The biomechanical effects of stretching. The American Journal of Sports Medicine, 18, 300-309. http://dx.doi.org/10.1177/036354659001800314

[18] Dutton, M. (2004) The Response of Biological Tissue to Stress. In: Brown, M. and Davis, K., Eds., Orthopaedic Examination, Evaluation, and Intervention, McGraw-Hill, New York, 87-100.

[19] Ehsan, A., Huang, J.I., Lyons, M. and Hanel, D.P. (2012) Surgical Management of Posttraumatic Elbow Arthrofibrosis. Journal of Trauma and Acute Care Surgery, 72, 1399-1403.

[20] Weizenbluth, M., Eichenblat, M., Lipskeir, E. and Kessler, I. (1989) Arthrolysis of the Elbow. 13 Cases of Posttraumatic Stiffness. Acta Orthopaedica Scandinavica, 60, 642-645. http://dx.doi.org/10.3109/17453678909149594 
[21] Bruno, R.J., Lee, M.L., Strauch, R.J. and Rosenwasser, M.P. (2002) Posttraumatic Elbow Stiffness: Evaluation and Management. Journal of the American Academy of Orthopaedic Surgeons, 10, 106-116.

[22] Marti, R., Kerkhoffs, G., Maas, M. and Blankevoort, L. (2002) Progressive Surgical Release of a Posttraumatic Stiff Elbow: Technique and Outcome after 2-18 Years in 46 Patients. Acta Orthopaedica, 73, 144-150. http://dx.doi.org/10.1080/000164702753671713

[23] Jupiter, J.B., O’Driscoll, S.W. and Cohen, M.S. (2003) The Assessment and Management of the Stiff Elbow. Instructional Course Lectures, 52, 93-111.

[24] Charalambous, C.P. and Morrey, B.F. (2012) Posttraumatic Elbow Stiffness. The Journal of Bone \& Joint Surgery, 94, 1428-1437.http://dx.doi.org/10.2106/JBJS.K.00711 